\title{
Isolation of Plasmopara viticola from Grapevine Leaves $^{\dagger}$
}

\author{
Camelia Ungureanu 1,*, Liliana Cristina Soare ${ }^{2}$, Diana Vizitiu ${ }^{3}$, Mirela Calinescu 4 , \\ Irina Fierascu ${ }^{5}$ and Radu Claudiu Fierascu ${ }^{5}$ \\ 1 Departament of General Chemistry, University "Politehnica" of Bucharest, Faculty of Applied Chemistry \\ and Materials Science 1-7, Polizu Str., 011061 Bucharest, Romania \\ 2 University of Pitesti, 1 Targul din Vale Street, 110040 Pitesti, Argeș County, Romania; soleil_cri@yahoo.com \\ 3 National Research and Development Institute for Biotechnology in Horticulture Stefanesti Arges, Stefanesti \\ City, Soseaua Bucharest - Pitesti Street, No 37, Arges 117715, Romania; vizitiud@yahoo.com \\ 4 Research Institute for Fruit Growing Pitesti - Maracineni, 117450 Maracineni, 402 Marului Str., Arges \\ County 110006, Romania; elacalinescu@yahoo.com \\ 5 National Institute for Research \& Development in Chemistry and Petrochemistry - ICECHIM Bucharest, \\ 202 Spl. Independentei 060021, Bucharest, Romania; dumitriu.irina@yahoo.com (I.F.); \\ radu_claudiu_fierascu@yahoo.com (R.C.F.) \\ * Correspondence: ungureanucamelia@gmail.com \\ + Presented at the 15th International Symposium "Priorities of Chemistry for a Sustainable Development" \\ PRIOCHEM, Bucharest, Romania, 30th October-1st November 2019.
}

Published: 14 October 2019

Keywords: Plasmopara viticola; grapevine; isolation; biofungicides

In order to test some biofungicides, the isolation of Plasmopara viticola was carried out. Plasmopara viticola is a fungus that causes the grapevine downy mildew disease [1,2].

The fungus strain was cultivated onto potato dextrose agar (abbreviated "PDA") from Sigma-Aldrich with next composition: agar, $15 \mathrm{~g} / \mathrm{L}$, dextrose, $20 \mathrm{~g} / \mathrm{L}$, and potato extract, $4 \mathrm{~g} / \mathrm{L}$. A chloramphenicol antibiotic was used to avoid bacterial contamination. Experiences were effectuated with samples (leaves) from the National Research and Development Institute for Biotechnology in Horticulture Stefanesti, Arges.

Morphological observations (Figure 1) were taken based on colony, conidia and conidiophore morphology, and other morphological characters [3].

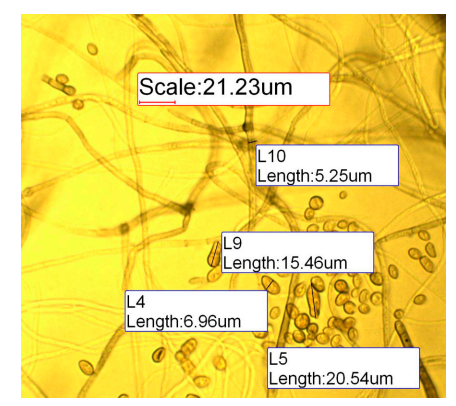

Figure 1. Plasmopara viticola (microscope view)-sporangiophores and sporangia. Identification according to $[2,3]$.

Plasmopara viticola was isolated from infected grapevine leaves and grown on the potato dextrose agar culture medium with the goal to test some biofungicides. 
Acknowledgments: This work was supported by a grant of the Romanian Ministry of Research and Innovation, CCCDI-UEFISCDI, project number PN-III-P1-1.2-PCCDI-2017-0332"/Project 3, contract 6PCCDI/2018, within PNCDI II.

\section{References}

1. Sawant, I.S.; Wadkar, P.N.; Ghule, S.B.; Rajguru, Y.R.; Salunkhe, V.P.; Sawant, S.D. Enhanced biological control of powdery mildew in vineyards by integrating a strain of Trichoderma afroharzianum with sulphur. Biol. Control. 2017, 114, 133-143.

2. Black, G.M.E.; Gold, R.E.; Düggelin, M.; Guggenheim, R. Mycol. Res. 1997, 101, 1033-1046.

3. Agrios, G.N. Plant Pathology, 5th ed.; Elsevier-Academic Press: San Diego, CA, USA, 2005.

(C) 2019 by the authors. Licensee MDPI, Basel, Switzerland. This article is an open access article distributed under the terms and conditions of the Creative Commons Attribution (CC BY) license (http://creativecommons.org/licenses/by/4.0/). 